\title{
SPECTRUM SLIDING ANALYSIS
}

\author{
Vladimir Ya. Krakovsky \\ vladimir_krakovsky@hotmail.com \\ National Aviation University \\ Kiev, Ukraine
}

\begin{abstract}
The Spectrum Sliding Analysis (SSA) is a dynamic spectrum analysis in which the next analysis interval differs from the previous one by including the next signal sample and excluding the first one from the previous analysis interval. Such a spectrum analysis is necessary for time-frequency localization of analyzed signals with given peculiarities. Using the well-known Fast Fourier Transform (FFT) towards this aim is not effective. Recursive algorithms which use only one complex multiplication for computing one spectrum sample during each analysis interval are more effective. The author improved one algorithm so that it is possible to use only one complex multiplication for computing two, four, and even eight (for complex signals) spectrum samples simultaneously. Problems of realization and application of the spectrum sliding analysis are also considered in the chapter.
\end{abstract}

keywords: spectrum sliding analysis, dynamic spectrum analysis, recursive algorithms, sliding spectrum algorithms \& analyzers, instant spectrum algorithms \& analyzers, algorithms for instantaneous spectrum digital analyzers, multichannel filter

\section{Introduction}

Depending on the form of signal presentation, SSA may be implemented by analog, digital, or discrete-analog spectrum analyzers. Narrow band analog filters can be used to implement SSA at some points of the frequency's working band. However, such analyzers are usually designed to analyze power spectrums and are not capable of analyzing phase spectrum and complex spectrum Cartesian constituents, which restricts their application [7]. The spectrum analysis discrete-analog method uses the discrete signal formed by sampling the signal to be analyzed with an uncrossed pulse sequence, the magnitudes of which are altered in accordance with the sample values. This type of analyzer permits spectrum analysis with quality and quantity compatibility conditions, satisfying the information completeness condition 
[7], and it may be adapted for SSA [8, 9]. Spectrum digital analyzers [1] are the most popular. There are two SSA spectrum methods: in one, the origin of the reference function is matched with the interval analysis origin. This approach computes the sliding spectrum [10]. In some applications (e.g., speech recognition) there is no need for such matching. When this is the case the computation is called the instant spectrum [10]. Algorithms and devices implementing the two methods are considered in the following sections.

\section{Sliding Spectrum Algorithms and Devices}

The discrete sliding spectrum is given by the formula:

$$
F_{q}(p)=\frac{1}{N} \sum_{k=q+N-1}^{q} f(k) W_{N}^{-p(k-(q-N+1))}, p \in \overline{0, P-1}, q=0,1,2, \ldots
$$

where $q$ is an analysis interval index, $k$ is a signal sample index in the limits of the sliding window $k \in \overline{q-N+1, q}, N$ is the size of the processing extract, $F_{q}(p)$ is the complex spectrum sample at the frequency $p \Delta \omega$ at the instant $q \Delta t, f(k)$ is the analyzed signal sample value at the instant $k \Delta t$ and $W_{N}^{-p k}$ is the complex reference coefficient symbolic representation $\exp \left(-j \frac{2 \pi}{N} p k\right) . \Delta t$ and $\Delta \omega$ are the discrete time and frequency intervals, determined by the information completeness condition [7].

Direct computation of the functional (1) requires $N P$ complex multiplications and $(N-1) P$ additions. FFT [11] reduces this number by $\frac{N}{\log _{2} N}$. Further reduction is possible. The formula:

$$
\begin{aligned}
F_{q}(p)=\left(F_{q-1}(p)+\frac{1}{N}(f(q)-f(q-N))\right) W_{N}^{p}, & p \in \overline{0, P-1}, \\
q & =0,1,2, \ldots .
\end{aligned}
$$

leads to a recursive algorithm [12] computing the sliding spectrum samples. The computer requirements (number of complex multiplications) are considerably lower using (2) rather than (1). In (1), $N$ complex multiplications are computed for each spectrum sample $\left(\log _{2} N\right.$ if the FFT is used). Using (2) the same computation requires only one complex multiplication. This advantage has led to many sliding spectrum analyzers implementing (2).

The spectrum analyzer functional diagram implementing the algorithm (2) is shown in Fig. 1. The following symbols are used in the diagram: 
$\mathrm{ADC}$ is the analog-digital converter, $\mathrm{DR}$ is the delay register, $\mathrm{SU}$ is the subtraction unit, AD is the adder, WU is the weighting unit, MU is the multiplier, RAM is the random-access memory; $f(t)$ is the analog input signal; $f_{q}$ are digital readouts of the signal $f(t) ; f_{q-N}$ are the readouts $f_{q}$ delayed by $N$ steps; $\Delta f_{q}$ are the readouts of the difference signal $f_{q}-f_{q-N}$; $W_{N}^{p}$ are the readouts of the weighting function $\exp \left(j \frac{2 \pi}{N} p\right) ; F_{q}(p), F_{q-1}(p)$ are the sliding spectrum readouts in the current and the preceding steps, respectively.

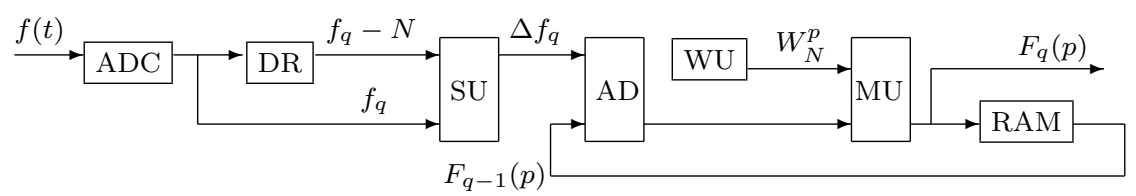

Figure 1. The spectrum analyzer functional diagram (2)

A detailed description of the characteristics of the analyzers [13-15], implementing (2), are given in [16]. Computational stability using algorithm (2) is achieved by the appropriate rounding of twiddle factor constituents having factor modulus not exceeding unity [17]. The constituents word size should be half of the sliding steps number binary logarithm [18]. For an unlimited number of sliding steps it is possible to use the technical decision described in [19]. While using algorithm (2) the reference function is always matched with the analysis interval origin, thereby ensuring a matched filtering [20-22].

\section{Instant Spectrum Algorithms and Devices}

The instant spectrum

$$
F_{q}(p)=\frac{1}{N} \sum_{k=q-N+1}^{q} f(k) W_{N}^{-p k}, \quad p \in \overline{0, P-q}, \quad q=0,1,2, \ldots
$$

can be implemented by the following simple recursive algorithm, in [23, 24].

$$
F_{q}(p)=F_{q-1}(p)+\frac{1}{N}(f(q)-f(q-N)) W_{N}^{-p q}, p \in \overline{0, P-1}, q=0,1,2, \ldots
$$

This algorithm is always stable and the twiddle factors $W_{N}^{-p q}$ word size 
is the same as the number of signal samples. Moreover, it is possible to achieve matching with an additional multiplier and a complex conjugate device [10]. In $[25,26]$ devices that implement this algorithm using the conveyer mode are described.

The spectrum analyzer functional diagram implementing algorithm (4) is shown in Fig. 2, where $\Delta F_{q}(p)$ are the increments of the spectrum readouts $\left(\frac{1}{N}\right) \Delta f_{q} W_{N}^{-p q}$.

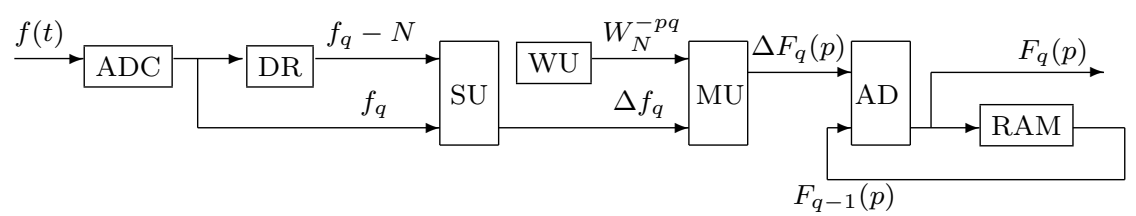

Figure 2. The spectrum analyzer functional diagram (4)

The spectrum analyzer functional diagram implementing algorithm (4) with matching is shown in Fig. 3, where CCU and AMU are the complex conjugate device and additional multiplier, respectively [10].

This algorithm has a remarkable property which permits one to organize SSA so that one complex multiplication may be used for computing two, four, and even eight (for complex signals) spectrum harmonics at once [3-6, 27]. This may be done by presenting algorithm (4) as follows:

$$
\begin{gathered}
F_{(p)}=F_{q-1}(p)+\Delta F_{q}(p), \quad p \in \overline{0, P-1}, \quad q=0,1,2, \ldots, \\
\Delta F_{q}(p)=\frac{1}{N}(f(q)-F(q-N)) \exp \left(-j \frac{2 \pi}{N} p q\right) .
\end{gathered}
$$

The spectrum increments $\Delta F_{q}(p)$ may be used not only for spectrum harmonic p, but also for the spectrum harmonics

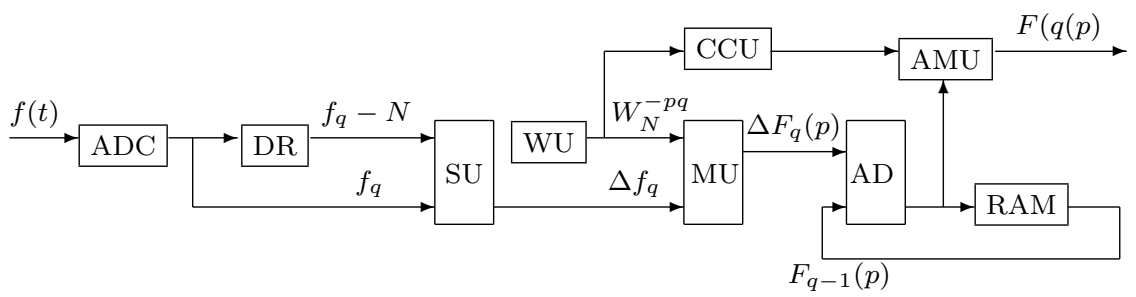

Figure 3. Spectrum analyzer function with matching 


$$
p_{i}=i \frac{N}{4}+p, i \in \overline{1,3}
$$

and

$$
p_{k}=k \frac{N}{4}-p, k \in \overline{1,4}
$$

using known properties of the complex exponential function. A simplified summary of the algorithm (5) can be described as follows:

a) for harmonics of the form (7)

$$
F_{q}\left(p_{i}\right)=F_{q-1}\left(p_{i}\right)+(-j)^{i q} \Delta F_{q}(p), \quad q=0,1,2, \ldots,
$$

b) for harmonics of the form (8)

$$
F_{q}\left(p_{k}\right)=f_{q-1}\left(p_{k}\right)+(-j)^{k} q \Delta F_{q}(-p), \quad q=0,1,2, \ldots,
$$

where $F_{q}(-p)$ are complex conjugated spectrum increments $\Delta F_{q}(p)$, if the signal samples are real. If the signal samples are complex, the increments $\Delta F_{q}(-p)$ are generated by inverting the signs of the products of the signal increments $\Delta f_{q}=\frac{1}{N}(f(q)-f(q-N))$ with the imaginary part of the weighting function and then forming the appropriate algebraic sums.

Devices implementing algorithms (9) and (10) are presented in [2832]. Their generalized functional diagram is shown in Fig. 4, where SW are switching components, $m$ is the number of additional subranges, $m \in$ $\{1,3,7\}$.

In the simplest case of complex signal analysis $(P=N)$, the harmonic range is partitioned into two subranges $p \in \overline{0, \frac{N}{2}-1}$, and by (7) for $i=2$ we have $p_{2}=\frac{N}{2}+p$. By (9) the readouts for the harmonics $p_{2}$ are determined by:

$$
F_{q}\left(p_{2}\right)=F_{q-1}\left(p_{2}\right)+(-1)^{q} \Delta F_{q}(p) .
$$

i.e., the spectrum increment $\Delta F_{q}(p)$ for harmonic $p$ obtained at the $\mathrm{MU}$ output are fed through SW to the input of the adder for subrange $p_{2}$. In SW, as we see from (11), the sign of the increments $\Delta F_{q}(p)$ are either preserved or inverted, depending on the parity of the readout index $q$. To this end, it is sufficient to include in SW two operand sign control units (for the real and imaginary components of $\Delta F_{q}(p)$ ), which are controlled 


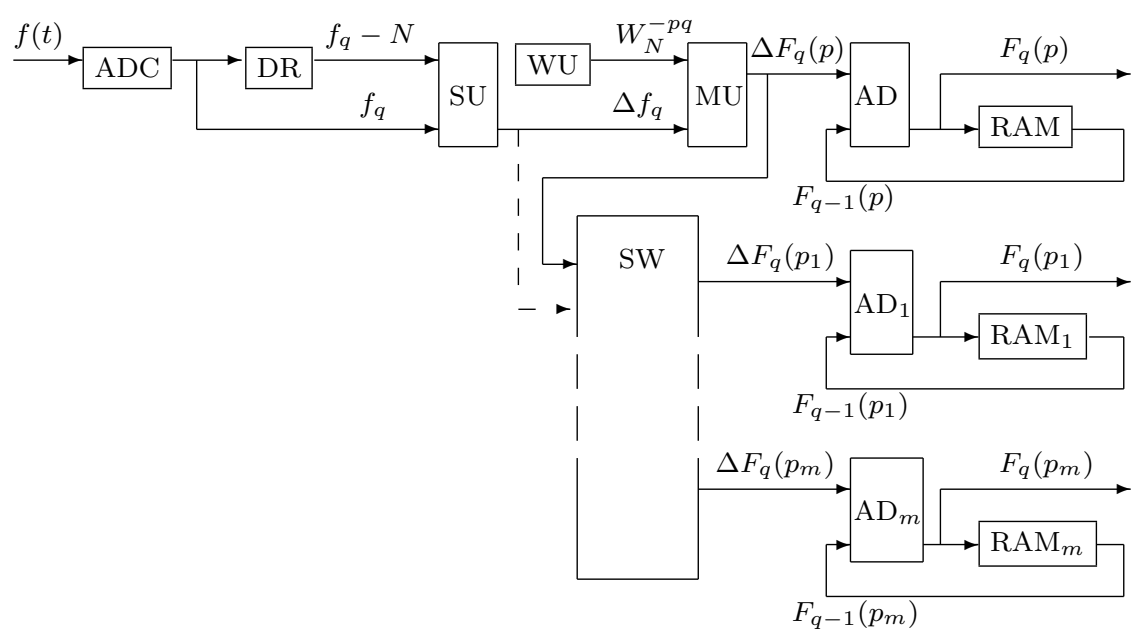

Figure 4. Generalized functional diagram: Implementation device

by the binary signal from the output of the least significant bit in the $q$-counter [28]. Operand sign control can be performed by exclusive OR circuits. Simultaneous evaluation of two harmonics doubles the functional speed at a small additional hardware cost.

Using (7) and dividing the harmonic range into four (for a complex signal) or two (for a real signal) subranges, we can similarly design appropriate analyzer structures $[29,30]$. In this case SW, in addition to operand sign control unit, also contains multiplexers for the operations $(-j)^{i} q \Delta F_{q}(p)$. The activation sequence of these multiplexers with the corresponding operand sign control circuits can be determined from the data in Table I, which gives the dependences of the increments $\Delta F_{q}\left(p_{1}\right), \Delta F_{q}\left(p_{2}\right)$, and $\Delta F_{q}\left(p_{3}\right)$ on $\Delta F_{q}(p)$ and $q$.

We see from Table I that the multiplexer control signal can be taken from the output of the least significant bit of the $q$-counter, while the control signals for the operand sign control circuits can be provided either by the outputs of the two least significant bits of this counter or by their logical XOR.

The SSA speed can be further doubled if, in addition to (7), we also use (8). In this case, SW must also include components that generate the spectrum increments $\Delta F_{q}(-p)(10)$. Moreover, the second SW input should receive from the $\mathrm{SU}$ the signal readout increment $\Delta F_{q}$ (dashed line in Fig. 4 ). This is needed for organizing simultaneous processing in corresponding subranges of operands of the harmonics $(2 i+1) \frac{N}{8}$ and $k \frac{N}{4}, i \in \overline{1,3}, k \in \overline{1,4}$. 
TABLE I. Multiplexer control signal

\begin{tabular}{|c|c|c|c|}
\hline $\begin{array}{c}q \\
\bmod \\
4\end{array}$ & $\Delta F_{q}\left(p_{1}\right)$ & $\Delta F_{q}\left(p_{2}\right)$ & $\Delta F_{q}\left(p_{3}\right)$ \\
\hline 0 & $\Delta F_{q}(p)$ & $\Delta F_{q}(p)$ & $\Delta F_{q}(p)$ \\
\hline 1 & $\operatorname{Im} \Delta F_{q}(p)-j \operatorname{Re} \Delta F_{q}(p)$ & $-\Delta F_{q}(p)$ & $-\operatorname{Im} \Delta F_{q}(p)+j \operatorname{Re} \Delta F_{q}(p)$ \\
\hline 2 & $-\Delta F_{q}(p)$ & $\Delta F_{q}(p)$ & $-\Delta F_{q}(p)$ \\
\hline 3 & $-\operatorname{Im} \Delta F_{q}(p)+j \operatorname{Re} \Delta F_{q}(p)$ & $-\Delta F_{q}(p)$ & $\operatorname{Im} \Delta F_{q}(p)-j \operatorname{Re} \Delta F_{q}(p)$ \\
\hline
\end{tabular}

To explain this technique, Tables II and III list the indices of simultaneously determined harmonics for real [31] and complex [32] signals. With the determination of the harmonic $p=\frac{N}{8}, \Delta F_{q}(p)$ is used to determine the harmonics $(2 i+1) \frac{N}{8}, i \in \overline{1,3}$, and $\Delta F_{q}$ is used at the same time to calculate the harmonics $k \frac{N}{4}, k \in \overline{1,4}$.

TABLE II. Indices of simultaneously determined harmonics

\begin{tabular}{|c|c|c|c|c|c|}
\hline Subrange & \multicolumn{5}{|c|}{ Indices of simultaneously determined harmonics } \\
\hline$p$ & 1 & 2 & $\cdots$ & $\frac{N}{8}-1$ & $\frac{N}{8}$ \\
\hline$p_{1}$ & $\frac{N}{4}-1$ & $\frac{N}{4}-2$ & $\ldots$ & $\frac{N}{8}+1$ & $\frac{N}{4}$ \\
\hline$p_{2}$ & $\frac{N}{4}+1$ & $\frac{N}{4}+2$ & $\cdots$ & $3 \frac{N}{8}-1$ & $3 \frac{N}{8}$ \\
\hline$p_{3}$ & $\frac{N}{2}-1$ & $\frac{N}{2}-2$ & $\ldots$ & $3 \frac{N}{8}+1$ & 0 \\
\hline
\end{tabular}

Structural diagrams of analyzers based on algorithms (9) and (10) are given in the invention descriptions [28-32]. These analyzers use modern hardware components and require some additional hardware costs to expand the frequency range by two, four, or eight times. The additional 
hardware costs increase more slowly than the incremental benefits produced by group complex multiplication.

TABLE III. Indices of simultaneously determined harmonics

\begin{tabular}{|c|c|c|c|c|c|}
\hline Subrange & \multicolumn{3}{|l|}{ Indices of simultaneously determined harmonics } \\
\hline$p$ & 1 & 2 & $\ldots$ & $\frac{N}{8}-1$ & $\frac{N}{8}$ \\
$p_{1}$ & $\frac{N}{4}-1$ & $\frac{N}{4}-2$ & $\ldots$ & $\frac{N}{8}+1$ & $\frac{N}{4}$ \\
$p_{2}$ & $\frac{N}{4}+1$ & $\frac{N}{4}+2$ & $\ldots$ & $3 \frac{N}{8}-1$ & $3 \frac{N}{8}$ \\
$p_{3}$ & $\frac{N}{2}-1$ & $\frac{N}{2}-2$ & $\ldots$ & $3 \frac{N}{8}+1$ & $\frac{N}{2}$ \\
$p_{4}$ & $\frac{N}{2}+1$ & $\frac{N}{2}+2$ & $\ldots$ & $5 \frac{N}{8}-1$ & $5 \frac{N}{8}$ \\
$p_{5}$ & $3 \frac{N}{4}-1$ & $3 \frac{N}{4}-2$ & $\ldots$ & $5 \frac{N}{8}+1$ & $3 \frac{N}{4}$ \\
$p_{6}$ & $3 \frac{N}{4}+1$ & $3 \frac{N}{4}+2$ & $\ldots$ & $7 \frac{N}{8}-1$ & $7 \frac{N}{8}$ \\
$p_{7}$ & $N-1$ & $N-2$ & $\ldots$ & $7 \frac{N}{8}+1$ & 0 \\
\hline
\end{tabular}

\section{Multichannel Matched Filtering on the Basis of SSA}

SSA may be used for FIR-filtering [33] in invariant to information shift (stationary) mode. Such filters perform signal convolution with a given impulse response

$$
s(\tau)=\int_{\tau-T}^{\tau} f^{*}(t) g(t-\tau) d t=\int_{0}^{T} f^{*}(t+\tau) g(t) d t .
$$

In accordance with Parseval's theorem (12) is equivalent to the integral

$$
s(\tau)=\frac{1}{2 \pi} \int_{-\infty}^{\infty} F_{\tau}(\omega) G^{*}(\omega) d \omega,
$$


where $G(\omega)$ is the unshifted impulse response spectrum, and $F_{\tau}(\omega)$ is the signal sliding spectrum in the analysis interval $T$. The discrete version of (13) is

$$
r(q)=\sum_{p=0}^{P-1} F_{q}(p) G^{*}(p),
$$

where $F_{q}(p)$ and $G^{*}(p)$ are samples of the spectra $F_{\tau}(\omega)$ and $G^{*}(\omega), P$ is the number of impulse response spectrum readouts. Expression (14) is equivalent to the traditional discrete correlation

$$
r(q)=\sum_{k=0}^{N-1} f(k+q) g(k) .
$$

The expression (14) is the formal description of the FIR-filtering algorithm on the basis of SSA [22]. Practical realization of the filtration procedure while processing with a set of $D$ different impulse responses may be performed by the device, whose functional diagram is shown in Fig. 5, where SSA is a harmonic sliding analyzer; $\mathrm{MU}_{i}$ are multipliers, $i \in 1, D$; $\mathrm{ROM}_{-} \mathrm{G}^{*}{ }_{i}$ are complex conjugated transfer function ROMs of the spectral readouts for the appropriate filter channels; and $\mathrm{AA}_{i}$ are accumulating adders of the pair products.

In such a device mutually overlapped input signal readouts series are used, with the help of SSA, to continuously in time complete the sliding spectrum readouts series $F_{q}(p), q=0,1,2, \ldots$. These values are simultaneously placed in all the $\mathrm{MU}_{i}$. The outputs of the $\mathrm{MU}_{i}$ are connected to second inputs of the ROM_G ${ }_{i}{ }_{i}$, which, synchronously with each $p$-th harmonic readout of the sliding spectrum, present respective $p$-th harmonic readouts of the complex conjugated transfer function spectrum. The products from the $\mathrm{MU}_{i}$ outputs are placed into the $\mathrm{AA}_{i}$ inputs. At the end of each sliding step, we form the sum (14) of the readouts $r_{i}(q)$.

As follows from Fig. 5, each channel of filtering consists of one accumulating adder, one ROM_ $\mathrm{G}_{i}{ }_{i}$, and one multiplier connected to the common output of the SSA. The channel structure is uniform throughout. The difference is determined by the contents of the transfer function spectral readouts ROM_G ${ }_{i}{ }$.

The concrete performances of the appropriate devices for multichannel filtering on the basis of SSA are given in [20] for real signals and [21] for complex signals.

A criterion of effectiveness of the multichannel filter based on the SSA is the number of complex multiplications used for realization of one step of sliding on the set of $D$ channels of filtering. In accordance with algorithm (14) and the structure of the complex multichannel filter the number $\alpha_{s}$ 


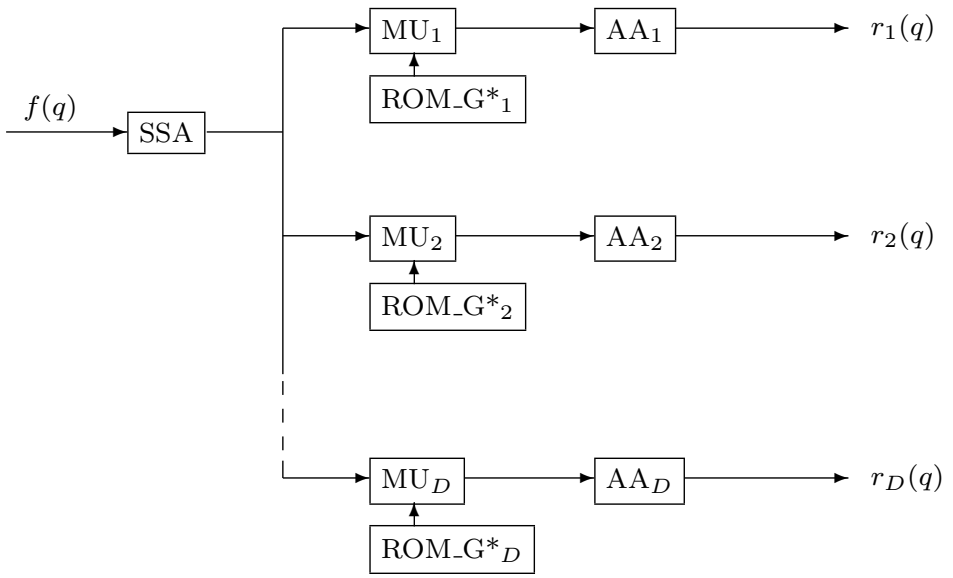

Figure 5. Varying impulse responses

of complex multiplication operations realizing one step of sliding is determined by the sum of the operations in the first stage of processing (stage of computing the sliding spectrum) and operations in the second stage of processing ( $D$ times of weighting and summation of the spectral components). Thus,

$$
\alpha_{s}=P+D P=P(1+D) .
$$

The appropriate computing expenditure realizing the standard correlation procedure of the complex multichannel filtering (15) is $\alpha_{c}=D N$.

Consequently, the benefit of the effectiveness of the multichannel filtering on the basis of SSA can be estimated by the ratio of the computing expenditures

$$
B=\frac{\alpha_{c}}{\alpha_{s}}=\frac{D N}{P(1+D)} .
$$

The more $N$ and the less $P$, compared with $N$, the more advantageous is filtering on the basis of SSA. It is possible to show that, in the case of matched (optimal) filtering, the number $P$ practically coincides with the base of the useful signal $Q=\Delta F T$. The most benefit is realized in the use of matched filtering when $P=1$. In this case the filter degenerates into $D$ independent digital resonators. If $N>>D$, then

$$
B_{\max }=\frac{D N}{1+D} \approx N .
$$


As the order of the realized filter or base of the filtered signal increases $(P$ increases) the relative benefit decreases. In the limit, when $P=N$

$$
B_{\min }=\frac{D}{1+3} \approx 1,
$$

and the effectiveness of the digital filter on the basis of SSA does not essentially differ from the effectiveness of the correlation filter.

It is important to note that the decrease in computing expenditures, when using SSA, leads to an almost proportional decrease in the hardware volume used. For example, when realizing $D$-channel filtering on the basis of correlation convolution (15) it is necessary to use $D$ multipliers and adders working in parallel. The speed of each is determined by the necessity to perform $N$ multiplications and additions for one sliding step. When realizing multichannel filters on the basis of SSA the number of multipliers and adders used to achieve the same productivity may be decreased approximately by $N / P$ times, because each channel performs only $P$ multiplications. In the extreme case when organizing $D$ digital resonators $(P=1)$ it is sufficient to use solely the multiplier in the SSA, since the secondary processing in each channel completely degenerates and the SSA itself serves as the multichannel filter.

When filtering simple and low complexity signals in conditions of high degree a priori frequency uncertainty, when $N>>P$, multichannel filter on the basis of SSA has advantages in hardware expenditures not only in comparison with correlation FIR-filtering but also with very effective filtering on the basis of FFT [33].

Multichannel filtering devices contain a direct Fourier transform unit. The output is connected with $D$ filtering channels, each of which consists of a weighting unit and an inverse Fourier transform unit. These devices, without special buffer memory units, form the readouts of filtering the signal irregularly in time. This distorts the time scale and the regularity of the output information. To achieve stationary mode using these devices, we require additional hardware expenditures. Furthermore, the presence in each channel of an inverse Fourier transform unit increases weight, volume, and cost of the hardware. Devices [20, 21], which realize the discussed algorithm of filtration, are free of these drawbacks.

\section{Recommendation on Spectrum Sliding Analysis Realization}

It is also possible to increase the response speed of the instant digital spectrum analyzers using table multiplication [34]. This may be used if there is enough memory for appropriate tables. 
An A-D converter with the aim to increase the accuracy may be used. A converter [35] with Sample-Hold circuit [36] allows an increase in both the accuracy and the speed. Project [37] showed that it is possible to achieve an accuracy of 16 bits with the sampling frequency up to $2 \mathrm{MHz}$. Projects [38] and [39] showed that using Xilinxs PLAs Virtex-2 [40] it is possible to implement inventions [31] and [32] with the sampling frequency up to 3.9 $\mathrm{MHz}$ for the size of processing extracts $\mathrm{N}=256$ and the accuracy of 8 bits.

Spectrum digital analyzers output only Cartesian constituents of the spectrum complex samples. If only the modulus of these samples is required, it is possible to increase the response speed by using approximating methods of modulus calculations based on comparison, shift, and summation [41, 42].

\section{Acknowledgments}

The author would like to thank Myoung An, Richard Tolimieri, and Benjamin Shenefelt for their help in preparing this chapter for publication.

All the material presented in this paper has been reproduced from the text Spectrum Sliding Analysis, Vladimir Ya. Krakovsky, Psypher Press, 2006, with explicit permissions from the author and the publisher.

\section{References}

[1] V.N. Plotnikov, A.V. Belinsky, V.A. Sukhanov and Yu.N. Zhigulevtsev, Spectrum digital analyzers. Moscow: Radio i svjaz, 1990. (In Russian).

[2] R. Tolimieri, M. An, Time-Frequency Representations. Birkhauser, 1998.

[3] V.Ya. Krakovsky, "Algorithms for increase of speed of response for digital analyzers of the instant spectrum". Kibernetika, (5), pp.113-115, 1990. (In Russian).

[4] V.Ya. Krakovskii. Generalized representation and implementation of speed-improvement algorithms for instantaneous spectrum digital analyzers. Cybernetics and System Analysis, 32, (4), pp.592-597, March 1997.

[5] V.Ya. Krakovsky. "Spectrum Sliding Analysis Algorithms \& Device". Proceedings of the 1st International Conference on Digital Signal Processing and Its Application, June 30 - July 3, 1998, I, pp.104-107, Moscow, Russia. [6] V.Ya.Krakovsky. "Harmonic Sliding Analysis Problem". Twentieth Century Harmonic Analysis - A Celebration, Ed. James S. Byrnes, NATO Science Series, II. Mathematics, Physics and Chemistry, 33, pp. 375-377, 2000.

[7] V.I. Chajkovsky, "Spectrum experimental analysis metrology peculiarities". Kiev, 1977. (Preprint/Ukraine SSR NAS Institute of Cybernetics; 77-6). (In Russian). 
[8] V.I. Chajkovsky and V.Ya. Krakovsky, "Sliding spectrum discrete-analog analyzer". USSR Author Certificate 666488, June 5, 1979.

[9] V.Ya.Krakovsky, "Instant spectrum discrete-analog analyzer". USSR Author Certificate 834576, May 30, 1981.

[10] V.I. Chajkovsky, "Real time spectrum digital analyzers functional peculiarities". Kiev, 1976. (Preprint/Ukraine SSR NAS Institute of Cybernetics; 76-39). (In Russian).

[11] J.W.Cooley and J.W.Tukey, "An algorithm for the machine calculation of complex Fourier series". Math.Comput., 19, pp.297-301, 1965.

[12] J.H. Halberstein, "Recursive, complex Fourier analysis for real-time applications". Proc.IEEE, 54, p.903, 1966.

[13] V.I. Chajkovsky, V.F. Koval, V.Ya. Krakovsky and V.S. Pikulin, "Fourier spectrum analyzer". USSR Author Certificate 560232, May 30, 1977.

[14] V.I. Chajkovsky, V.Ya. Krakovsky and .F. Koval, "Fourier spectrum digital analyzer". USSR Author Certificate 614440, July 5, 1978.

[15] G. M. Dillard, "Method and apparatus for computing the discrete Fourier transform recursively". USA Patent 4023028, May 10, 1977.

[16] V.Ya. Krakovsky, "Development and investigation of specialized computing devices for electrical signals spectrum sliding analysis". Eng. Sci. Cand. Dissertation, NAS Institute of Cybernetics, Kiev, 1980. (In Russian). [17] V.Ya. Krakovsky and V.I. Chajkovsky, "Spectrum sliding analysis peculiarities". Autometrija, 6, pp.34-37, 1984. (In Russian).

[18] V.Ya. Krakovsky, "Selection of sliding spectrum digital analyzer twiddle factors word size". Izmeritelnaja tekhnika, 10, pp.13-14, 1983. (In Russian).

[19] D.A.Perreault and T.C.Rich, "Method and apparatus for suppression of error accumulation in recursive computation of a discrete Fourier transform". USA Patent 4225937, Sep. 30, 1980.

[20] V.I. Chajkovsky and V.Ya. Krakovsky, "Multichannel filter". USSR Author Certificate 995282, Feb. 7, 1983.

[21] V.I. Chajkovsky and V.Ya. Krakovsky, "Multichannel filter". USSR Author Certificate 1193778 A, Nov. 11, 1985.

[22] V.I. Chajkovsky and V.Ya. Krakovsky, "Signal discrete filtration on the basis of spectrum sliding analysis". Kibernetika $i$ vychislitelnaja tekhnika, (71), pp. 55-58. Kiev: Naukova dumka, 1986.

[23] R.D. Lejtes and V.N. Sobolev, Synthetic telephone systems digital modeling. Moscow: Svjaz, 1969. (In Russian).

[24] J.W.Schmitt and D.L.Starkey, "Continuously updating Fourier coefficients every sampling interval". USA Patent 3778606, Dec.11, 1973.

[25] V.Ya. Krakovsky and V.F. Koval, "Instant spectrum digital analyzer". USSR Author Certificate 932419, May 30, 1982.

[26] V.Ya. Krakovsky and V.F. Koval, "Instant spectrum digital analyzer". 
USSR Author Certificate 1095093 A, May 30, 1984.

[27] V.Ya. Krakovskii, "Digital analyzers for dynamic spectral analysis". Meas. Tech., USA, 36, (12), p.1324-30. Translation of: Izmer.Tekh. (Russia), 36, (12), pp.13-16. (Dec.1993).

[28] V.Ya. Krakovsky and V.F. Koval, "Instant spectrum digital analyzer". USSR Author Certificate 1377762 A2, Feb. 29, 1988.

[29] V.Ya. Krakovsky and V.F. Koval, "Complex signal instant spectrum digital analyzer". USSR Author Certificate 1406507 A2, June 30, 1988.

[30] V.Ya. Krakovsky and V.F. Koval, "Instant spectrum digital analyzer". USSR Author Certificate 1456904 A2, Feb. 7, 1989.

[31] V.Ya. Krakovsky, V.F. Koval, Z.A. Kuznetsova, and V.V. Eresko, "Real signal instant spectrum digital analyzer". USSR Author Certificate 1563408 A2. Permission for publication N 267/22, 28.11.91.

[32] V.Ya. Krakovsky, V.F. Koval, Z.A. Kuznetsova and V.V. Eresko, "Complex signal instant spectrum digital analyzer". USSR Author Certificate 1568732 A2. Permission for publication N 267/22, 28.11.91.

[33] L.R.Rabiner, B.Gold, Theory and application of digital signal processing. Prentice-Hall, Inc., Englewood Cliffs, New Jersey, 1975.

[34] V.N. Sobolev, "Dynamic spectral analysis fast algorithm". All-Union ARSO-16 seminar reports abstracts, pp. 232-233. Moscow State University, 1991. (In Russian).

[35] V.I. Chajkovsky, V.Ya. Krakovsky, and V.F. Koval, "A-D converter". USSR Author Certificate 1401608 A2, May 7, 1988.

[36] V.Ya. Krakovsky, "Sample-Hold circuit". USSR Author Certificate 1185398 A, Oct. 15, 1985.

[37] L.S. Litovchenko, "Computer system for precise measurement of analog signals: Diploma project". Project leader V.Ya. Krakovsky. Kyiv: National Aviation University, 2003. (In Ukrainian).

[38] P.V. Sergienko, "Real signal instant spectrum digital analyzer in the information processing systems: Diploma project". Project leader V.Ya. Krakovsky. Kyiv: National Aviation University, 2003. (In Ukrainian).

[39] S.V. Smetaniuk, "Complex signal instant spectrum digital analyzer in the information processing systems: Diploma project". Project leader V.Ya. Krakovsky. Kyiv: National Aviation University, 2003. (In Ukrainian). [40] http://www.xilinx.com

[41] V.Ya. Krakovsky, "Complex quantities modulus approximate determination". Metrology, (4), pp. 7-10, 1983. (In Russian).

[42] V.Ya. Krakovsky, "Complex quantities modulus approximate determination errors". Metrology, (4), pp. 25-30, 1992. (In Russian). 